\title{
Weak type estimates for certain Calderón-Zygmund singular integral operators
}

by

\author{
Atanas Stefanov (Amherst, MA)
}

\begin{abstract}
We prove weak type $(1,1)$ estimates for a special class of CalderónZygmund homogeneous kernels represented as $l^{1}$ sums of "equidistributed" $H^{1}$ atoms on $\mathbb{S}^{1}$.
\end{abstract}

1. Introduction. Let $\Omega: \mathbb{S}^{n-1} \rightarrow \mathbb{C}$ be a complex-valued integrable function, with mean zero. We consider the Calderón-Zygmund operator defined by

$$
T_{\Omega} f(x)=\lim _{\varepsilon \rightarrow 0} \int_{|y|>\varepsilon} \frac{\Omega(y /|y|)}{|y|^{n}} f(x-y) d y .
$$

The $L^{p}$ boundedness of $T_{\Omega}$ was proved by Calderón-Zygmund [1] under the optimal size condition

$$
\int_{\mathbb{S}^{n-1}}|\Omega(\theta)| \log ^{+}|\Omega(\theta)| d \theta<\infty .
$$

The question of weak type $(1,1)$ boundedness is more involved (the $L^{p}$ case being an easy consequence). Hofmann [6] (when $\Omega \in L^{q}, q>1, n=2$ ) and Christ-Rubio de Francia [3] (when $\Omega \in L \log ^{+}(L), n \leq 7$ ) proved weak type bounds for the operator $T_{\Omega}$. It is worth mentioning that the ideas for these results originated in [2], where similar estimates for the corresponding maximal function were obtained. Finally, Seeger [7] proved a weak type $(1,1)$ result under condition (1) in all dimensions. While as we indicated, (1) is an optimal size condition, it is far from being necessary. In fact, Coifman and Weiss [4] and independently Connett [5] proved $L^{p}$ boundedness of $T_{\Omega}$ under the more general assumption $\Omega \in H^{1}\left(\mathbb{S}^{n-1}\right)$ (see also [8]). This condition reflects the size as well as the oscillation of the kernel. We will be concerned here about the following

2000 Mathematics Subject Classification: Primary 42B20.

Key words and phrases: Calderón-Zygmund singular integrals, $H^{1}$ kernels, weak type estimates. 
Problem. What is the most general assumption on $\Omega$ such that the operator $T_{\Omega}$ is of weak type $(1,1)$, i.e.

$$
\left\|T_{\Omega} f\right\|_{L^{1, \infty}} \leq C\|f\|_{L^{1}} ?
$$

In particular does (2) hold if $\Omega \in H^{1}\left(\mathbb{S}^{n-1}\right)$ ?

We answer this question affirmately for a special class of $H^{1}$ kernels on $\mathbb{S}^{1}$. Let

$$
\Omega(\theta)=\sum_{\nu=1}^{N} \lambda_{\nu} a_{\nu}(\theta),
$$

where $\lambda_{\nu}>0$ and $a_{\nu}$ are $H^{1}\left(\mathbb{S}^{1}\right)$ atoms. The usual definition of $H^{1}\left(\mathbb{S}^{1}\right)$ requires that $\|\Omega\|_{H^{1}} \sim \sum \lambda_{\nu}$. We consider a special class of $H^{1}$ kernels by imposing an extra condition on the structure of the atoms, namely we want them to be of comparable size and have "almost" disjoint supports. To be precise, let $I_{\nu}$ be the support of $a_{\nu}$ and $e_{\nu}$ be the center of the arc $I_{\nu}$. For simplicity we assign increasing numbers counterclockwise and assume that $e_{1}=(1,0)$. We assume that there exists $l \in \mathbb{N}$ (which will be fixed throughout the argument) such that

(1) $\left|a_{\nu}(\theta)\right| \leq 2^{l}$,

(2) $\left|I_{\nu}\right| \sim 2^{-l}$,

(3) $\left|I_{\nu} \cap I_{\nu^{\prime}}\right| \leq \frac{1}{2} \min \left\{\left|I_{\nu}\right|,\left|I_{\nu^{\prime}}\right|\right\}$.

Condition (3) above can be rewritten as $\left|e_{\nu}-e_{\nu^{\prime}}\right| \geq c_{0}\left|\nu-\nu^{\prime}\right| 2^{-l}$ and the constant $c_{0}$ will be implicitly present in most of our estimates. In particular $N \leq 2 \pi 2^{l} / c_{0}$. We are now ready to state our main result.

THEOREM 1. If $\Omega=\sum_{\nu=1}^{N} \lambda_{\nu} a_{\nu}$ as above, then

$$
\left\|T_{\Omega} f\right\|_{L^{1, \infty}} \leq C\|f\|_{L^{1}} \sum_{\nu} \lambda_{\nu}
$$

where the constant $C$ is independent of $N, l, f$.

REMARKs. $\bullet \lambda_{\nu}>0$ is not a restriction of generality, because we can always incorporate modulus one numbers in the atoms.

- The $L \log ^{+} L$ theory is largely inapplicable even for single $H^{1}$ atoms. The reason is that the proofs never make use of the oscillation properties of the kernel. In a sense, $T_{\Omega} f$ is a bilinear operator acting on $\Omega$ and $f$, thus we ought to decompose $f$ taking into consideration the structure of $\Omega$.

Let us introduce some notations and conventions. We always denote the Lebesgue measure of some set $A$ by $|A|$. If $Q$ is a rectangle, then $Q^{*}$ will be used to denote the rectangle with the same center and three times longer sides. All the sums are with respect to every index present in the sums, unless otherwise indicated. 
In Section 2 we present a variant of the standard Calderón-Zygmund decomposition needed for our purposes. In Section 3 we prove Theorem 1 basing on three lemmas dealing with $L^{2}$ and $L^{1}$ estimates for the operator. In the last two sections we demonstrate the technical lemmas used to prove Theorem 1.

2. Calderón-Zygmund decompositions. Fix $\lambda>0$ and a function $f$. We will apply the standard decomposition $f=g+b$, where $g$ always stands for a "good" part and $b$ for a "bad" part. In our case, we shall need different decompositions in all directions $\left\{e_{\nu}\right\}_{\nu=1}^{N}$. This way, one gets $N$ different decompositions, which later on will be used to analyze the operator $T_{\Omega}$.

For every $\nu \in\{1, \ldots, N\}$, let $A_{\nu}$ be the set of all rectangles with one long side parallel to $e_{\nu}$ with length $\delta$ and a shorter side of length $\delta 2^{-l}$. Define the uncentered directional maximal function in direction $e_{\nu}$ by

$$
M_{\nu} f(x)=\sup _{x \in Q \in A_{\nu}} \frac{1}{|Q|} \int_{Q}|f(y)| d y .
$$

Since the doubling constant for the family $A_{\nu}$ is independent of $l$, we have

$$
\left\|M_{\nu} f\right\|_{L^{1, \infty}} \leq C\|f\|_{1},
$$

where $C$ is independent of $l$. Define an exceptional set $E_{\nu}=\left\{x:\left|M_{\nu} f(x)\right|>\right.$ $\left.\lambda / \lambda_{\nu}\right\}, \nu=1, \ldots, N$. Note that $\left|E_{\nu}\right| \leq\|f\|_{1} \lambda_{\nu} / \lambda$ by (3). We proceed as follows:

- Represent $E_{\nu}$ as $\bigcup_{i} Q_{\nu, i}$, where $Q_{\nu, i} \in A_{\nu}$ are disjoint and $Q_{\nu, i}^{*} \cap E_{\nu}^{\mathrm{c}} \neq \emptyset$ for every $\nu$, i.e.

$$
\int_{Q_{\nu, i}}|f(y)| d y \leq C \frac{\lambda}{\lambda_{\nu}}\left|Q_{\nu, i}\right| .
$$

Define

$$
\begin{aligned}
& b_{Q_{\nu, i}}(x)=\left(f(x)-\frac{1}{\left|Q_{\nu, i}\right|} \int_{Q_{\nu, i}} f(y) d y\right) \chi_{Q_{\nu, i}}(x), \\
& g_{Q_{\nu, i}}(x)=\frac{1}{\left|Q_{\nu, i}\right|}\left(\int_{Q_{\nu, i}} f(y) d y\right) \chi_{Q_{\nu, i}}(x) .
\end{aligned}
$$

- Let $J_{\nu}=\left\{x: M f(x)>\lambda /\left(\sum \lambda_{\nu}\right)\right\} \cup \bigcup_{k \neq \nu} E_{k} \backslash E_{\nu}$. Represent $J_{\nu}$ as $\bigcup_{Q_{\nu, i} \in A_{\nu}} Q_{\nu, i}$, where $\left\{Q_{\nu, i}\right\}$ are disjoint, and define $b_{Q_{\nu, i}}$ and $g_{Q_{\nu, i}}$ as above.

- For $K=\mathbb{R}^{2} \backslash\left(\bigcup E_{\nu} \cup\left\{x: M f(x)>\lambda /\left(\sum \lambda_{\nu}\right)\right\}\right)$, set $g(x)=f(x)$ for $x \in K_{\nu}$ and $g(x)=0$ otherwise.

Therefore for every $\nu \in\{1, \ldots, N\}$ we get a decomposition

$$
f(x)=\sum_{i} b_{Q_{\nu, i}}+\sum_{i} g_{Q_{\nu, i}}+g=\sum_{i} b_{Q_{\nu, i}}+g_{\nu}+g
$$


where

(1) $\int b_{Q_{\nu, i}}=0$,

(2) $\int\left|b_{Q_{\nu, i}}\right| \leq C\left(\lambda / \lambda_{\nu}\right)\left|Q_{\nu, i}\right|, \sum_{i}\left\|b_{Q_{\nu, i}}\right\|_{1} \leq C\|f\|_{1}$,

(3) $\sum\left|Q_{\nu, i}\right| \leq \sum\left|E_{\nu}\right|+\left|\left\{x: M f(x)>\lambda /\left(\sum \lambda_{\nu}\right)\right\}\right| \leq C \sum \lambda_{\nu}\|f\|_{1} / \lambda$,

(4) $\left\|g_{\nu}\right\|_{\infty} \leq \lambda / \lambda_{\nu},\left\|g_{\nu}\right\|_{1} \leq\|f\|_{1}$,

(5) $\|g\|_{\infty} \leq \lambda /\left(\sum \lambda_{\nu}\right),\|g\|_{1} \leq\|f\|_{1}$.

Without loss of generality we may and do assume that the sides of the selected cubes are of dyadic sidelength. We will denote by $d(Q)$ the sidelength of the long side of $Q$.

3. Proof of Theorem 1. For fixed $\lambda, f$, we have

$$
\begin{aligned}
\left|\left\{x:\left|T_{\Omega} f(x)\right|>\lambda\right\}\right| \leq & \left|\left\{x:\left|T_{\Omega} g(x)\right|>\lambda / 3\right\}\right| \\
& +\left|\left\{x:\left|\sum_{\nu} \lambda_{\nu} \frac{a_{\nu}}{|x|^{2}} * g_{\nu}(x)\right|>\lambda / 3\right\}\right| \\
& +\left|\left\{x:\left|\sum_{\nu} \sum_{i} \lambda_{\nu} \frac{a_{\nu}}{|x|^{2}} * b_{Q_{\nu, i}}(x)\right|>\lambda / 3\right\}\right| .
\end{aligned}
$$

Clearly

$$
\left|\left\{x:\left|T_{\Omega} g(x)\right|>\lambda / 3\right\}\right| \leq \frac{9}{\lambda^{2}}\left\|T_{\Omega}\right\|_{2}^{2}\|g\|_{2}^{2} \leq C \frac{\sum \lambda_{\nu}}{\lambda}\|f\|_{1},
$$

which takes care of the first term. The second term is relatively easy to handle via $L^{2}$ estimates (see Lemma 1 ). The third term on the right hand side of (4) requires further considerations. Let $\beta: \mathbb{R}^{+} \rightarrow \mathbb{R}$ be a smooth function such that

$$
\operatorname{supp} \beta \subset(1 / 2,2) \text { and } \sum_{j \in \mathbb{Z}} \beta\left(2^{-j} t\right)=1 \quad \text { for all } t>0 .
$$

Then

$$
K(x)=\frac{\Omega(x)}{|x|^{2}}=\sum_{\nu, j} \lambda_{\nu} a_{j, \nu}(x),
$$

where $a_{j, \nu}=\left(a_{\nu}(x) /|x|^{2}\right) \beta\left(2^{-j}|x|\right)$. Introduce also smooth functions $\psi, \Phi$ : $\mathbb{R} \rightarrow \mathbb{R}$ such that

(1) $\operatorname{supp} \psi \subset(-1,1)$ and $\psi(t)=1$ for $t \in(-1 / 2,1 / 2)$,

(2) $\operatorname{supp} \Phi \subset(-2,-1 / 2) \cup(1 / 2,2)$

and

$$
\sum_{m \geq 0} \Phi\left(2^{-m} t\right)+\psi(t)=1 \quad \text { for all } t \in \mathbb{R}
$$


Define $B_{j, \nu}=\sum_{d\left(Q_{\nu, i}\right)=2^{j}} b_{Q_{\nu, i}}$. Thus the third term on the right of (4) is controlled by

$$
\begin{aligned}
\mid\left\{x: \mid \sum_{\nu} \sum_{Q_{\nu, i}} \lambda_{\nu}\right. & \left.\frac{a_{\nu}}{|x|^{2}} * b_{Q_{\nu, i}}(x) \mid>\lambda / 3\right\} \mid \\
\leq & \left|\left\{x:\left|\sum_{j} \sum_{s>0} \sum_{\nu} \lambda_{\nu} a_{j, \nu} * B_{j-s, \nu}(x)\right|>\lambda / 6\right\}\right| \\
& +\left|\left\{x:\left|\sum_{j} \sum_{s \leq 0} \sum_{\nu} \lambda_{\nu} a_{j, \nu} * B_{j-s, \nu}(x)\right|>\lambda / 6\right\}\right| .
\end{aligned}
$$

The support of the function in the second expression on the right hand side of (6) is in a multiple of the exceptional set $\bigcup_{\nu} E_{\nu}$ and therefore by the properties of the decomposition we obtain the desired estimate for it. For the first expression on the right hand side of (6) we will need a further decomposition of the kernel. Let $P_{\nu}^{s}$ be a function so that $\widehat{P_{\nu}^{s}}(\xi)=\psi\left(2^{l-s / 4}\left\langle\xi /|\xi|, e_{\nu}\right\rangle\right)$. Convolution with $P_{\nu}^{s}$ essentially restricts the Fourier transform of the function to the part of the sphere "almost" orthogonal to $e_{\nu}$, as hinted by the uncertainty principle.

We split the first term on the right of (6) in two parts as follows:

$$
\begin{aligned}
& \left|\left\{x:\left|\sum_{j} \sum_{s>0} \sum_{\nu} \lambda_{\nu}\left(a_{j, \nu} * B_{j-s, \nu}\right)(x)\right|>\lambda / 6\right\}\right| \\
& \leq\left|\left\{x:\left|\sum_{j} \sum_{s>0} \sum_{\nu} \lambda_{\nu}\left(a_{j, \nu} * P_{\nu}^{s} * B_{j-s, \nu}\right)(x)\right|>\lambda / 12\right\}\right| \\
& \quad+\left|\left\{x:\left|\sum_{j} \sum_{s>0} \sum_{\nu} \lambda_{\nu}\left(a_{j, \nu} *\left(\delta-P_{\nu}^{s}\right) * B_{j-s, \nu}\right)(x)\right|>\lambda / 12\right\}\right|
\end{aligned}
$$

It is convenient to handle (4) and the first term on the right of (7) using $L^{2}$ estimates and the second term on the right of (7) using an $L^{1}$ estimate. We need the following lemmas.

Lemma 1. Let $\left\{a_{\nu}\right\}$ be a collection of atoms of comparable size and almost disjoint supports as in the statement of Theorem 1. If $\left\|g_{\nu}\right\|_{2}^{2} \leq$ $\lambda\|f\|_{1} / \lambda_{\nu}$, then

$$
\left\|\sum_{\nu} \lambda_{\nu}\left(\frac{a_{\nu}(\cdot)}{|\cdot|^{2}} * g_{\nu}\right)\right\|_{2}^{2} \leq C \lambda\left(\sum \lambda_{\nu}\right)\|f\|_{1} .
$$

Lemma 2. Let $b_{Q_{\nu, i}}$ be the family of functions obtained in the CalderónZygmund decomposition and

$$
B_{j, \nu}=\sum_{Q_{\nu, i}: d\left(Q_{\nu, i}\right)=2^{j}} b_{Q_{\nu, i}} .
$$


Then for every $s>0$, we have

$$
\left\|\sum_{j} \sum_{\nu} \lambda_{\nu}\left(a_{j, \nu} * P_{\nu}^{s} * B_{j-s, \nu}\right)\right\|_{2}^{2} \leq C 2^{-s / 4} \lambda\|f\|_{1} \sum \lambda_{\nu} .
$$

Finally, we state the $L^{1}$ estimate needed to estimate the second term in (7).

Lemma 3. Let $\left\{a_{\nu}\right\}$ be a family of atoms as above. Then

$$
\left\|a_{j, \nu} *\left(\delta-P_{\nu}^{s}\right)\right\|_{1} \leq C 2^{-s / 8}
$$

The proof can be easily finished assuming the validity of these three lemmas. First, since $\left\|g_{\nu}\right\|_{2}^{2} \leq\left\|g_{\nu}\right\|_{\infty}\left\|g_{\nu}\right\|_{1} \leq \lambda\|f\|_{1} / \lambda_{\nu}$, by Lemma 1 we have

$$
\begin{aligned}
\left|\left\{x:\left|\sum_{\nu} \lambda_{\nu}\left(\frac{a_{\nu}(\cdot)}{|\cdot|^{2}} * g_{\nu}\right)(x)\right|>\lambda / 3\right\}\right| & \leq \frac{9}{\lambda^{2}}\left\|\sum_{\nu} \lambda_{\nu} \frac{a_{\nu}(\cdot)}{|\cdot|^{2}} * g_{\nu}\right\|_{2}^{2} \\
& \leq C \frac{\sum \lambda_{\nu}}{\lambda}\|f\|_{1} .
\end{aligned}
$$

Next, Lemma 2 yields

$$
\begin{aligned}
\mid\left\{x: \mid \sum_{j} \sum_{s>0}\right. & \left.\sum_{\nu} \lambda_{\nu}\left(a_{j, \nu} * P_{\nu}^{s} * B_{j-s, \nu}\right)(x) \mid>\lambda / 12\right\} \mid \\
\leq & \frac{144}{\lambda^{2}}\left(\sum_{s>0}\left\|\sum_{j} \sum_{\nu} \lambda_{\nu}\left(a_{j, \nu} * P_{\nu}^{s} * B_{j-s, \nu}\right)\right\|_{2}\right)^{2} \\
\leq & \frac{C}{\lambda^{2}}\left(\sum_{s>0} 2^{-s / 8}\left(\lambda\|f\|_{1} \sum \lambda_{\nu}\right)^{1 / 2}\right)^{2} \leq \frac{C}{\lambda}\|f\|_{1} \sum \lambda_{\nu} .
\end{aligned}
$$

Finally, by Lemma 3 we have

$$
\begin{aligned}
\mid\left\{x: \mid \sum_{j} \sum_{s>0} \sum_{\nu}\right. & \left.\lambda_{\nu}\left(a_{j, \nu} *\left(\delta-P_{\nu}^{s}\right) * B_{j-s, \nu}\right)(x) \mid>\lambda / 12\right\} \mid \\
\leq & \frac{C}{\lambda} \sum_{j} \sum_{s>0} \sum_{\nu} \lambda_{\nu}\left\|a_{j, \nu} *\left(\delta-P_{\nu}^{s}\right)\right\|_{1}\left\|B_{j-s, \nu}\right\|_{1} \\
\leq & \frac{C}{\lambda} \sum_{s>0} 2^{-s / 8} \sum_{j} \sum_{\nu} \lambda_{\nu}\left\|B_{j-s, \nu}\right\|_{1} \leq \frac{C}{\lambda}\|f\|_{1} \sum_{\nu} \lambda_{\nu} .
\end{aligned}
$$

This concludes the proof of the theorem based on the lemmas.

4. $L^{2}$ estimates. Consider the disjoint decomposition of $\mathbb{R}^{2}$ into the cones

$$
A_{\nu}=\left\{\xi \in \mathbb{R}^{2}:(\nu-1) 2^{-l} \leq\left|\left\langle e_{1}, \xi /|\xi|\right\rangle\right| \leq \nu 2^{-l}\right\}, \quad \nu=1, \ldots, 2^{l} .
$$

We will need the following claim. 
Claim 1. If $a_{\nu}$ is an $H^{1}\left(\mathbb{S}^{1}\right)$ atom supported in $I_{\nu}$ with $\left\|a_{\nu}\right\|_{\infty} \leq 2^{l}$, $\left|I_{\nu}\right|=2^{-l}$, then for every $\xi \in A_{\nu^{\prime}}, \nu^{\prime} \neq \nu$, we have

$$
\left|\mathcal{F}\left(a_{\nu}(\cdot) /|\cdot|^{2}\right)(\xi)\right| \leq C /\left(1+\left|\nu-\nu^{\prime}\right|\right),
$$

where $\mathcal{F}$ denotes the Fourier transform.

Proof. According to the explicit formula for the Fourier transform of homogeneous kernels (see [10], p. 39), we have

$$
\mathcal{F}\left(a_{\nu}(\cdot) /|\cdot|^{2}\right)(\xi)=\int_{\mathbb{S}^{1}} a_{\nu}(\theta)\left[\ln \left(\frac{1}{|\langle\theta, \xi /|\xi|\rangle|}\right)+\frac{\pi i}{2} \operatorname{sign}\langle\theta, \xi\rangle\right] d \theta
$$

The above formula makes sense at least in the case $a_{\nu} \in H^{1}\left(\mathbb{S}^{1}\right)$ and the estimate by a constant is immediate (via the $H^{1}$-BMO duality). In particular, the $L^{2}$ norm of the corresponding convolution operator is uniformly bounded. Since $\int a_{\nu}=0$, it is easy to see that if $\xi \in A_{\nu^{\prime}}, \nu^{\prime} \neq \nu$, then

$$
\begin{aligned}
\left|\mathcal{F}\left(a_{\nu}(\cdot) /|\cdot|^{2}\right)(\xi)\right| & \leq\left|\int_{\mathbb{S}^{1}} a_{\nu}(\theta)\left[\ln \left(\frac{1}{|\langle\theta, \xi /|\xi|\rangle|}\right)-\ln \left(\frac{1}{\left|\left\langle e_{\nu}, \xi /|\xi|\right\rangle\right|}\right)\right] d \theta\right| \\
& \leq \int\left|a_{\nu}(\theta)\right| \ln \left(1+\frac{\left\langle\theta-e_{\nu}, \xi /|\xi|\right\rangle}{\left\langle e_{\nu}, \xi /|\xi|\right\rangle}\right) d \theta \leq \frac{C}{1+\left|\nu-\nu^{\prime}\right|}
\end{aligned}
$$

since $\left|e_{\nu}-\theta\right| \leq 2^{-l}$ and $\left|\left\langle e_{\nu}, \xi /|\xi|\right\rangle\right| \sim\left|\nu-\nu^{\prime}\right| 2^{-l}$.

We are now prepared for the proof of Lemma 1.

Proof of Lemma 1. We use the function $P_{\nu}^{0}$ defined by

$$
\widehat{P_{\nu}^{0}}(\xi)=\psi\left(2^{l}\left\langle\xi /|\xi|, e_{\nu}\right\rangle\right)
$$

Clearly

$$
\begin{aligned}
\left\|\sum_{\nu} \lambda_{\nu} \frac{a_{\nu}(\cdot)}{|\cdot|^{2}} * g_{\nu}\right\|_{2}^{2} \leq & 2\left\|\sum_{\nu} \lambda_{\nu} \frac{a_{\nu}(\cdot)}{|\cdot|^{2}} * P_{\nu}^{0} * g_{\nu}\right\|_{2}^{2} \\
& +2\left\|\sum_{\nu} \lambda_{\nu} \frac{a_{\nu}(\cdot)}{|\cdot|^{2}} *\left(\delta-P_{\nu}^{0}\right) * g_{\nu}\right\|_{2}^{2}
\end{aligned}
$$

By support considerations, $\widehat{P_{\nu}^{0}} \widehat{P_{\nu^{\prime}}^{0}} \neq 0$ only if $\left|\nu-\nu^{\prime}\right| \leq 100 / c_{0}$. Therefore by Plancherel's theorem and Claim 1,

$$
\begin{aligned}
\left\|\sum_{\nu} \lambda_{\nu} \frac{a_{\nu}(\cdot)}{|\cdot|^{2}} * P_{\nu}^{0} * g_{\nu}\right\|_{2}^{2} & \leq C \sum_{\nu} \lambda_{\nu}^{2}\left\|\frac{a_{\nu}(\cdot)}{|\cdot|^{2}} * g_{\nu}\right\|_{2}^{2} \\
& \leq C \sum_{\nu} \lambda_{\nu}^{2}\left\|g_{\nu}\right\|_{2}^{2} \leq C\left(\sum \lambda_{\nu}\right) \lambda\|f\|_{1} .
\end{aligned}
$$


For the second term on the right (8) we use Claim 1, Plancherel's theorem, and Cauchy-Schwarz to obtain

$$
\begin{aligned}
& \left\|\sum_{\nu} \lambda_{\nu} \frac{a_{\nu}(\cdot)}{|\cdot|^{2}} *\left(\delta-P_{\nu}^{0}\right) * g_{\nu}\right\|_{2}^{2} \\
& \leq \sum_{\nu} \sum_{\nu^{\prime}} \lambda_{\nu} \lambda_{\nu^{\prime}} \int\left|\widehat{g}_{\nu}\right|\left|\widehat{g}_{\nu^{\prime}}\right|\left|\widehat{\frac{a_{\nu}(\cdot)}{|\cdot|^{2}}}\right|\left|\frac{a_{\nu^{\prime}}(\cdot)}{|\cdot|^{2}}\right|\left|1-\widehat{P_{\nu}^{0}}\right|\left|1-\widehat{P_{\nu^{\prime}}^{0}}\right| d \xi \\
& \leq C \sum_{\nu, \nu^{\prime}, \nu^{\prime \prime}} \lambda_{\nu} \lambda_{\nu^{\prime}} \int_{A_{\nu^{\prime \prime}}} \frac{\left|\widehat{g}_{\nu}\right|}{1+\left|\nu^{\prime \prime}-\nu^{\prime}\right|} \cdot \frac{\left|\widehat{g}_{\nu^{\prime}}\right|}{1+\left|\nu^{\prime \prime}-\nu\right|} d \xi \\
& \leq C \sum_{\nu, \nu^{\prime \prime}} \sum_{\nu^{\prime}} \lambda_{\nu}^{2} \int_{A_{\nu^{\prime \prime}}} \frac{\left|\widehat{g}_{\nu}\right|^{2}}{\left(1+\left|\nu^{\prime \prime}-\nu^{\prime}\right|\right)^{2}} d \xi \\
& +C \sum_{\nu, \nu^{\prime \prime}} \sum_{\nu} \lambda_{\nu^{\prime}}^{2} \int_{A_{\nu^{\prime \prime}}} \frac{\left|\widehat{g}_{\nu^{\prime}}\right|^{2}}{\left(1+\left|\nu^{\prime \prime}-\nu\right|\right)^{2}} d \xi \\
& \leq C \sum_{\nu} \lambda_{\nu}^{2}\left\|g_{\nu}\right\|_{2}^{2} \leq\left(\sum \lambda_{\nu}\right) \lambda\|f\|_{1} \text {. }
\end{aligned}
$$

We turn to the estimate concerning the first term on the right of (8). We use the $T T^{*}$ tools elaborated in [3], but note that no cancellation of the atoms $a_{\nu}$ is needed in the proof, whereas the cancellation of $b_{Q_{\nu, i}}$ is heavily exploited.

Proof of Lemma 2. Observe that since $\left\{e_{\nu}\right\}$ are "equidistributed", each $\xi$ is contained in no more than $C 2^{s / 4}$ sets of the form supp $\widehat{P_{\nu}^{s}}$. By Plancherel's identity,

$$
\begin{aligned}
\left\|\sum_{j} \sum_{\nu} \lambda_{\nu} a_{j, \nu} * P_{\nu}^{s} * B_{j-s, \nu}\right\|_{2}^{2} & =\left\|\sum_{j} \sum_{\nu} \lambda_{\nu} \widehat{a}_{j, \nu} \widehat{P}_{\nu}^{s} \widehat{B}_{j-s, \nu}\right\|_{2}^{2} \\
& \leq \sum_{\nu} \lambda_{\nu}^{2} 2^{s / 4}\left\|\sum_{j} a_{j, \nu} * B_{j-s, \nu}\right\|_{2}^{2} .
\end{aligned}
$$

Therefore it suffices to prove the following inequality:

$$
\left\|\sum_{j} a_{j, \nu} * B_{j-s, \nu}\right\|_{2}^{2} \leq C 2^{-s / 2} \frac{\lambda}{\lambda_{\nu}} \sum_{j}\left\|B_{j-s, \nu}\right\|_{1}
$$

for every $\nu=1, \ldots, N$.

Without loss of generality $e_{\nu}=e_{1}=(1,0)$ and for simplicity we drop dependence on $\nu$ from our notation. All the rectangles that appear below 
will be assumed to be in the family $A_{1}$. We have

$$
\begin{aligned}
\left\|\sum_{j} a_{j} * B_{j-s}\right\|_{2}^{2} & \leq 2\left|\sum_{i} \sum_{i \leq j}\left\langle a_{j} * B_{j-s}, a_{i} * B_{i-s}\right\rangle\right| \\
& \leq 2 \sum_{j}\left\|B_{j-s}\right\|_{1} \sum_{i \leq j}\left\|\widetilde{a}_{j} * a_{i} * B_{i-s}\right\|_{\infty} .
\end{aligned}
$$

Thus it suffices to prove $\sum_{i \leq j}\left\|\widetilde{a_{j}} * a_{i} * B_{i-s}\right\|_{\infty} \leq C 2^{-s / 2} \lambda / \lambda_{1}$. By dilation, this is equivalent to the case $j=0, i \leq 0$. Write $\left(\widetilde{a}_{0} * a_{i}\right)(y)=\left\langle L_{i}^{y} a, \widetilde{a}\right\rangle$, where

$$
L_{i}^{y} a(\theta)=\int a\left(\frac{y-t \theta}{|y-t \theta|}\right) \frac{\beta(|y-t \theta|)}{|y-t \theta|^{2}} \cdot \frac{\beta\left(2^{-i} t\right)}{t} d t .
$$

We see that $\widetilde{a}_{0} * a_{i} * B_{i-s}(0)=\left\langle T_{i, s} a, \widetilde{a}\right\rangle$, where

$$
T_{i, s} a(\theta)=\int B_{i-s}(-y) L_{i}^{y} a(\theta) d y=\sum_{Q: d(Q)=2^{i-s}} \int b_{Q}(-y) L_{i}^{y} a(\theta) d y .
$$

Since $\|\widetilde{a}\|_{\infty} \leq 2^{l}$ and $|I|=|\operatorname{supp} \widetilde{a}| \leq 2^{-l}$, it suffices to show that

$$
\sum_{i \leq 0} \sup _{\theta \in I}\left|T_{i, s} a(\theta)\right| \leq C 2^{-s / 2} \lambda / \lambda_{1}
$$

since the general case for $\widetilde{a_{0}} * a_{i} * B_{i-s}(x)$ follows by translation invariance.

To prove (11), note first that $\operatorname{supp}_{y} L_{i}^{y} a(\theta) \subseteq\left\{y: 1 \leq|y| \leq 2,|y /| y\left|-e_{1}\right|\right.$ $\left.\leq 2^{1-l}\right\}$. Set $\delta=2^{-l-s / 2}$. Consider two cases.

In the first case, $1 \leq|y| \leq 2,|y /| y\left|-e_{1}\right| \leq \delta$. Split the rectangles accordingly in two groups:

$$
\begin{aligned}
\mathcal{C}_{i}^{\prime} & =\left\{Q \in A_{1}: d(Q)=2^{i-s}, Q \cap\left\{y: 1 \leq|y| \leq 2,|y /| y\left|-e_{1}\right| \leq \delta\right\} \neq \emptyset\right\}, \\
\mathcal{C}_{i}^{\prime \prime} & =A_{1} \backslash \mathcal{C}_{i}^{\prime} \cap\left\{Q: d(Q)=2^{i-s}\right\} .
\end{aligned}
$$

Since $\left\|L_{i}^{y} a\right\|_{\infty} \leq C 2^{l}$ we have

$$
\begin{aligned}
\sum_{i \leq 0} \sup _{\theta}\left|\sum_{Q \in \mathcal{C}_{i}^{\prime}} \int_{Q} b_{Q}(-y) L_{i}^{y} a(\theta) d y\right| & \leq \sum_{i \leq 0} \sum_{Q \in \mathcal{C}_{i}^{\prime}}\left\|b_{Q}\right\|_{1}\left\|L_{i}^{y} a\right\|_{\infty} \\
& \leq C 2^{l} \frac{\lambda}{\lambda_{1}} \sum_{i \leq 0} \sum_{Q \in \mathcal{C}_{i}^{\prime}}|Q| \leq C 2^{l} \frac{\lambda}{\lambda_{1}} \delta \\
& =C \frac{\lambda}{\lambda_{1}} 2^{-s / 2},
\end{aligned}
$$

which gives the estimate in the first case. 
To treat the second case, we need the following

Claim 2. For every $\theta \in \operatorname{supp} a, 1 \leq|y| \leq 2, \delta \leq|y /| y\left|-e_{1}\right| \leq 2^{-l}$, we have

$$
\left|\frac{\partial}{\partial \theta} L_{i}^{y} a(\theta)\right| \leq C 2^{l-i}, \quad\left|\frac{\partial}{\partial \theta^{\perp}} L_{i}^{y} a(\theta)\right| \leq C 2^{l-i} \delta^{-1}=C 2^{2 l+s / 2-i},
$$

where $\theta^{\perp} \in \mathbb{S}^{1},\left\langle\theta^{\perp}, \theta\right\rangle=0$, and $\partial / \partial \theta$ is the directional derivative in direction $\theta$.

Proof. By rotation invariance, it suffices to check the Claim for $\theta=e_{1}$, i.e.

$$
\left|\frac{\partial}{\partial y_{1}} L_{i}^{y} a\left(e_{1}\right)\right| \leq C 2^{l-i}, \quad\left|\frac{\partial}{\partial y_{2}} L_{i}^{y} a\left(e_{1}\right)\right| \leq C 2^{l-i} \delta^{-1} .
$$

Note that since $|y /| y\left|-e_{1}\right|>\delta$, we have $\left|y_{2}\right|>c \delta$. Following [3], we make the change of variables $\omega=\left(y-t e_{1}\right) /\left|y-t e_{1}\right|$ and differentiate (10) with respect to $y_{1}$. Introduce also the inverse Jacobi factor $J(\omega, y)=|d \omega / d t|=$ $\left|y_{2}\right| /\left|y-t e_{1}\right|^{2}$. We have

$$
\begin{aligned}
\frac{\partial}{\partial y_{1}} L_{i}^{y} a\left(e_{1}\right)= & \int a(\omega) \frac{\partial}{\partial y_{1}}\left(\frac{\beta\left(\left|y-t(\omega) e_{1}\right|\right)}{\left|y-t(\omega) e_{1}\right|^{2}} \cdot \frac{\beta\left(2^{-i} t(\omega)\right)}{t(\omega)}\right) \frac{1}{J(\omega, y)} d \omega \\
& -\int a(\omega) \frac{\beta\left(\left|y-t(\omega) e_{1}\right|\right)}{\left|y-t(\omega) e_{1}\right|^{2}} \cdot \frac{\beta\left(2^{-i} t(\omega)\right)}{t(\omega)} \cdot \frac{\partial J}{\partial y_{1}} \cdot \frac{1}{J^{2}(\omega, y)} d \omega .
\end{aligned}
$$

The first term on the right is clearly bounded by $2^{l-i}$ (by changing coordinates back to $t)$, for the second observe that $\left|\partial J / \partial y_{1}\right| /|J| \leq C /\left|y-t e_{1}\right| \leq C$, since $\left|y-t e_{1}\right| \sim 1$. This implies the bound $2^{l}$ for the second term above.

The second estimate in (12) follows by the same argument, once we observe that $\left|\partial J / \partial y_{2}\right| /|J| \leq C\left|y-t e_{1}\right|^{4} /\left|y_{2}\right| \leq C / \delta$.

We estimate the remaining terms as follows:

$$
\begin{aligned}
\left|\sum_{Q \in \mathcal{C}_{i}^{\prime \prime}} \int b_{Q}(-y)\left(L_{i}^{y} a\right)(\theta) d y\right| \leq & \sum_{Q \in \mathcal{C}_{i}^{\prime \prime}} \int\left|b_{Q}(-y)\right| \cdot\left|L_{i}^{y} a(\theta)-L_{i}^{y_{Q}} a(\theta)\right| d y \\
\leq & \sum_{Q \in \mathcal{C}_{i}^{\prime \prime}} \int\left|b_{Q}(-y)\right| \cdot\left|L_{i}^{y} a(\theta)-L_{i}^{y_{\theta}} a(\theta)\right| d y \\
& +\sum_{Q \in \mathcal{C}_{i}^{\prime \prime}} \int\left|b_{Q}(-y)\right| \cdot\left|L_{i}^{y_{\theta}} a(\theta)-L_{i}^{y_{Q}} a(\theta)\right| d y
\end{aligned}
$$

where $y_{Q}$ is the center of $Q$ and $y_{\theta}$ is such that $y_{\theta}-y$ is parallel to $\theta$, and $y_{\theta}-y_{Q}$ is parallel to $\theta^{\perp}$ and $\left|y_{\theta}-y_{Q}\right| \leq 2^{i-s-l},\left|y-y_{\theta}\right| \leq 2^{i-s}$. This is possible, because $\left|\theta-e_{1}\right| \leq 2^{-l}$. Thus, according to Claim 2 and the previous 
inequality, we have

$$
\begin{aligned}
& \left|\sum_{i \leq 0} \sum_{Q \in \mathcal{C}_{i}^{\prime \prime}} \int b_{Q}(-y) L_{i}^{y} a(\theta) d y\right| \\
& \quad \leq \sum_{i \leq 0} \sum_{Q \in \mathcal{C}_{i}^{\prime \prime}}\left\|b_{Q}\right\|_{1}\left(\sup _{y}\left|\frac{\partial}{\partial \theta} L_{i}^{y} a(\theta)\right|\left|y-y_{\theta}\right|+\sup _{y}\left|\frac{\partial}{\partial \theta^{\perp}} L_{i}^{y} a(\theta)\right|\left|y_{\theta}-y_{Q}\right|\right) \\
& \quad \leq \lambda \sum_{i \leq 0} \sum_{Q \in \mathcal{C}_{i}^{\prime \prime}}|Q|\left(2^{i+l-s}+2^{i-s / 2+l}\right) / \lambda_{1} \leq C 2^{-s / 2} \lambda / \lambda_{1} .
\end{aligned}
$$

5. $L^{1}$ estimates. In this section we prove Lemma 3 . The methods are similar to those in [7], although we need one more decomposition in the angular variable. In what follows, the mean value zero property of the atoms will be crucial for us.

Proof of Lemma 3. Without loss of generality, it suffices to consider the case $e_{\nu}=(1,0)$. Using the construction of the function $\Phi$ in $(5)$, we write

$$
a_{j, 1}-P_{1}^{s} * a_{j, 1}=\sum_{m=1}^{l-s / 4+10} \mathcal{F}^{-1}\left(\Phi\left(2^{l-s / 4-m}\left\langle\xi /|\xi|, e_{1}\right\rangle\right)\right) * a_{j, 1},
$$

where $\mathcal{F}^{-1}$ is the inverse Fourier transform. Introduce also a function $\gamma \in$ $C_{0}^{\infty}$ with supp $\gamma \subseteq(1 / 2,2)$ such that $\sum \gamma^{2}\left(2^{-k} \xi\right)=1$. Define $\widehat{L}_{k}(\xi)=$ $\gamma\left(2^{-k} \xi\right)$. Thus

$$
a_{j, 1}-P_{1}^{s} * a_{j, 1}=\sum_{k, m} \mathcal{F}^{-1}\left[c_{j, k}^{m, s}\right] * L_{k}
$$

where

$$
c_{j, k}^{m, s}(\xi)=\gamma\left(2^{-k} \xi\right) \Phi\left(2^{l-s / 4-m}\left\langle\xi /|\xi|, e_{1}\right\rangle\right) \widehat{a}_{j, 1}(\xi) .
$$

Next, we claim that

$$
\left\|L_{k} * \mathcal{F}^{-1}\left(\Phi\left(2^{l-s / 4-m}\left\langle\xi /|\xi|, e_{1}\right\rangle\right)\right)\right\|_{1} \leq C
$$

uniformly with respect to $m, k, s$. To show this, observe that the following Sobolev embedding formula holds:

$$
\|\widehat{h}\|_{1} \leq C \sum_{|\alpha| \leq 2}\left\|\partial^{\alpha} h_{Q}\right\|_{2},
$$

where $h_{Q}(x):=h(Q x)$ and $Q$ is a nonsingular linear transformation. Consider then a linear transformation $A_{k, m}$ such that

$$
\xi=A_{k, m} \eta=\left(2^{k-l+s / 4+m} \eta_{1}, 2^{k} \eta_{2}\right) .
$$

To prove (13), it suffices to demonstrate

$$
\left\|\partial_{\eta}^{\alpha}\left[\widehat{L}_{k} \Phi\left(2^{l-s / 4-m}\left\langle\cdot /|\cdot|, e_{1}\right\rangle\right)\left(A_{k, m} \eta\right)\right]\right\|_{2} \leq C_{\alpha}
$$


for every multiindex $\alpha \in \mathbb{N}^{2}$. It is not difficult to see that this is the case (see Lemma 2.2 in [7] for a similar calculation). Thus by (13) and since $\int a_{1}(\theta) d \theta=0$, we have

$$
\begin{aligned}
& \left\|\mathcal{F}^{-1}\left[c_{j, k}^{m, s}\right] * L_{k}\right\|_{1} \leq C\left\|L_{k} * a_{j, 1}\right\|_{1} \\
& \quad=\left.C \int\left|\int L_{k}(x-y) a_{1}(y) \beta\left(2^{-j}|y|\right)\right| y\right|^{-2} d y \mid d x \\
& \quad \leq C \iiint \sup _{\theta}\left|L_{k}(x-r \theta)-L_{k}\left(x-r e_{1}\right)\right| \cdot\left|a_{1}(\theta)\right| \beta\left(2^{-j} r\right) r^{-1} d r d \theta d x \\
& \quad \leq C 2^{j-l}\left\|\nabla L_{k}\right\|_{1} \leq C 2^{k+j-l} .
\end{aligned}
$$

For large $k$, we need a more refined estimate. Again by Sobolev embedding we have

$$
\begin{aligned}
\| \mathcal{F}^{-1}[ & \left.\Phi\left(2^{l-s / 4-m}\left\langle\xi /|\xi|, e_{1}\right\rangle\right) \gamma^{2}\left(2^{-k} \xi\right) \widehat{a}_{j, 1}\right] \|_{1} \\
& \leq \sum_{|\alpha| \leq 2}\left\|\frac{\partial}{\partial \eta^{\alpha}}\left[\Phi\left(2^{l-s / 4-m}\left\langle\cdot /|\cdot|, e_{1}\right\rangle\right) \gamma^{2}\left(2^{-k} \cdot\right) \widehat{a}_{j, 1}(\cdot)\left(A_{k, m} \eta\right)\right]\right\|_{2} .
\end{aligned}
$$

Observe that the function has compact support $\subset\{\eta:|\eta| \leq 10\}$ and therefore the derivatives of $\Phi$ and $\gamma$ with respect to $\eta$ are bounded by a universal constant. Furthermore, the $L^{2}$ norm is dominated by the $L^{\infty}$ norm. Notice also that if $\Phi\left(2^{l-s / 4-m}\left\langle\xi /|\xi|, e_{1}\right\rangle\right) \gamma\left(2^{-k} \xi\right) \neq 0$, then $\left|\left\langle\xi /|\xi|, e_{1}\right\rangle\right| \sim 2^{m+s / 4-l}$, $|\xi| \sim 2^{k}$ and thus $|\langle\xi, \theta\rangle| \sim 2^{m+k+s / 4-l}$ for all $\theta \in \operatorname{supp} a_{1}$, since $m, s>0$. Having made these observations, we proceed to bound the corresponding derivatives of $\widehat{a}_{j, 1}$. We have

$\widehat{a}_{j, 1}\left(A_{k, m} \eta\right)=\int a_{1}(\theta) \int r^{-1} \beta\left(2^{-j} r\right) \exp \left(-i r\left(\theta_{1} 2^{k-l+s / 4+m} \eta_{1}+\theta_{2} 2^{k} \eta_{2}\right)\right) d r d \theta$.

After differentiating $\alpha$ times in $\eta$ and integrating by parts three times (in $r$ ) we get

$$
\begin{aligned}
\frac{\partial}{\partial \eta^{\alpha}} \widehat{a}_{j, 1}\left(A_{k, m} \eta\right)= & \int a_{1}(\theta) \frac{1}{\left(-i\left\langle\theta, A_{k, m} \eta\right\rangle\right)^{3}} \int \partial_{r}^{3}\left(r^{-1} \beta\left(2^{-j} r\right)\right)(-i r)^{|\alpha|} \\
& \times\left(\theta_{1} 2^{k+m-l+s / 4}\right)^{\alpha_{1}}\left(\theta_{2} 2^{k}\right)^{\alpha_{2}} \exp \left(-i r\left\langle\theta, A_{k, m} \eta\right\rangle\right) d r d \theta .
\end{aligned}
$$

Thus, taking into account that $\left|\left\langle\theta, A_{k, m} \eta\right\rangle\right| \sim 2^{m+k+s / 4-l}$ and $\left|\theta_{2}\right| \leq 2^{-l}$ we obtain

$$
\left\|\frac{\partial}{\partial \eta^{\alpha}} \widehat{a}_{j, 1}\left(A_{k, m} \eta\right)\right\|_{\infty} \leq \frac{C}{2^{3(k+j-l+m+s / 4)}}\left(2^{j+k+m-l+s / 4}+2^{k+j-l}\right)^{|\alpha|} .
$$

Since $|\alpha| \leq 2$, we finally arrive at

$$
\left\|\mathcal{F}^{-1}\left[\Phi\left(2^{l-s / 4-m}\left\langle\xi /|\xi|, e_{1}\right\rangle\right) \gamma^{2}\left(2^{-k} \xi\right) \widehat{a}_{j, 1}\right]\right\|_{1} \leq \frac{C}{2^{k+j-l+m+s / 4}} .
$$

Thus, the needed estimate for large $k$ is proved. We now have to combine 
the two estimates to obtain the final statement of Lemma 3. We have

$$
\begin{aligned}
\left\|\sum_{k, m} \mathcal{F}^{-1}\left[c_{j, k}^{m, s}\right] * L_{k}\right\|_{1} \leq & \sum_{m=1}^{l-s / 4+10} \sum_{k: k+j-l \leq-s / 8-m / 2}\left\|\mathcal{F}^{-1}\left[c_{j, k}^{m, s}\right] * L_{k}\right\|_{1} \\
& +\sum_{m=1}^{l-s / 4+10} \sum_{k: k+j-l \geq-s / 8-m / 2}\left\|\mathcal{F}^{-1}\left[c_{j, k}^{m, s}\right] * L_{k}\right\|_{1} \\
\leq C & \sum_{m=1}^{l-s / 4+10} 2^{-s / 8-m / 2} \leq C 2^{-s / 8} .
\end{aligned}
$$

Acknowledgements. I am indebted to Loukas Grafakos for initiating me into this problem.

\section{References}

[1] A. P. Calderón and A. Zygmund, On singular integrals, Amer. J. Math. 78 (1956), 289-309.

[2] M. Christ, Weak type $(1,1)$ bounds for rough operators, Ann. of Math. 128 (1988), $19-42$.

[3] M. Christ and J.-L. Rubio de Francia, Weak type $(1,1)$ bounds for rough operators, II, Invent. Math. 93 (1988), 225-237.

[4] R. R. Coifman and G. Weiss, Extensions of Hardy spaces and their use in analysis, Bull. Amer. Math. Soc. 83 (1977), 569-645.

[5] W. C. Connett, Singular integrals near $L^{1}$, in: Proc. Sympos. Pure Math. 35, S. Wainger and G. Weiss (eds.), Part I, Amer. Math. Soc., 1979, 163-165.

[6] S. Hofmann, Weak $(1,1)$ boundedness of singular integrals with nonsmooth kernels, Proc. Amer. Math. Soc. 103 (1988), 260-264.

[7] A. Seeger, Singular integral operators with rough convolution kernels, J. Amer. Math. Soc. 9 (1996), 95-105.

[8] A. Stefanov, Characterizations of $H^{1}$ and applications to singular integral operators, Illinois J. Math. 44 (2000), 574-592.

[9] E. M. Stein, Harmonic Analysis: Real-Variable Methods, Orthogonality, and Oscillatory Integrals, Princeton Univ. Press, Princeton, NJ, 1993.

[10] - - Singular Integrals and Differentiability Properties of Functions, Princeton Univ. Press, Princeton, NJ, 1970.

Department of Mathematics and Statistics

University of Massachusetts

Lederle Graduate Research Tower

Amherst, MA 01003, U.S.A.

E-mail: stefanov@math.umass.edu 